\title{
68Ga-PSMA PET/CT in prostate cancer patients - patterns of disease, benign findings and pitfalls
}

Zohar Keidar $^{1,2^{*}}$ (D), Ronit Gill ${ }^{1}$, Elinor Goshen ${ }^{3,4}$, Ora Israel ${ }^{1,2}$, Tima Davidson ${ }^{5}$, Maryna Morgulis ${ }^{5}$, Natalia Pirmisashvili ${ }^{1}$ and Simona Ben-Haim ${ }^{6,7}$

\begin{abstract}
Background: 68Ga-PSMA PET/CT has an important role in assessment of prostate cancer patients with biochemical recurrence and is evolving in staging high- and intermediate risk disease. The aim of present study was to describe the metastatic patterns and frequency of involved sites of prostate cancer and to assess the incidence of benign Ga68-PSMA avid PET/CT findings in a large patient population.
\end{abstract}

Methods: 68Ga-PSMA PET/CT studies performed in two tertiary medical centers over a period of 24 months were retrospectively reviewed. The incidence and location of pathological 68Ga-PSMA avid foci, suspicious to represent malignancy, as well as those of unexpected benign foci of increased 68Ga-PSMA activity were documented and analyzed.

Results: There were 445 68Ga-PSMA studies in 438 men (mean age 72.4, range 51-92 years) with prostate cancer referred for biochemical failure $(n=270,61 \%)$, staging high-risk disease $(n=112,25 \%)$, response assessment $(n=30,7 \%)$, follow-up $(n=22,5 \%)$ and suspected bone metastases $(n=11,2 \%)$. 68Ga-PSMA avid disease sites were observed in 319 studies (72\%), in 181 studies (67\%) for biochemical recurrence, 94 studies for staging (84\%) $(p<0.05)$, in 22 studies for response assessment $(73 \%), 10$ follow up studies $(45 \%)$ and in five patients with suspected bone metastases (45\%). 68Ga-PSMA avid lesions were most commonly detected in the prostate $(n=193,43 \%)$, loco-regional spread $(n=51,11 \%)$, abdomino-pelvic nodes $(n=129,29 \%)$ and distant metastases $(n=158,36 \%)$, including bone metastases $(n=11,25 \%)$, distant lymphadenopathy $(n=29$, 7\%) and other organs $(n=18,4 \%)$. Distant 68Ga-PSMA-avid metastases were commonly seen in patients with biochemical recurrence $(14 / 21$ lesions), but were not seen in patient referred for staging $(p<0.013)$. There were 96 non-malignant 68Ga-PSMA avid foci in 81 studies, most common in reactive lymph nodes $(n=36$, $38 \%)$, nonmalignant bone lesions $(n=21,22 \%)$, thyroid nodules $(n=9,9 \%)$, ganglions $(n=9,9 \%)$ and lung findings $(n=8,8 \%)$.

Conclusion: The distribution of 68Ga-PSMA avid metastatic lesions is similar to data previously reported mainly from autopsy with comparable detection rates, indicating 68Ga-PSMA PET/CT is an accurate detection tool in patients with metastatic prostate cancer. If confirmed by further prospective studies 68Ga-PSMA PET/ $\mathrm{CT}$ should be included in the guidelines to evaluate disease extent in these patients.

Keywords: Prostate cancer, 68Ga-PSMA, PET/CT

\footnotetext{
* Correspondence: zohar@keidar.net

${ }^{1}$ Department of Nuclear Medicine, Rambam Health Care Campus, Haifa, Israel

${ }^{2}$ The Bruce Rappaport Faculty of Medicine, Technion - Israel Institute of

Technology, Haifa, Israel

Full list of author information is available at the end of the article
}

(c) The Author(s). 2018 Open Access This article is distributed under the terms of the Creative Commons Attribution 4.0 International License (http://creativecommons.org/licenses/by/4.0/), which permits unrestricted use, distribution, and reproduction in any medium, provided you give appropriate credit to the original author(s) and the source, provide a link to the Creative Commons license, and indicate if changes were made. The Creative Commons Public Domain Dedication waiver (http://creativecommons.org/publicdomain/zero/1.0/) applies to the data made available in this article, unless otherwise stated. 


\section{Introduction}

Prostate cancer is the most common solid malignancy in men and the 3rd leading cause of cancer related death with estimated 161,360 new cases and 6730 estimated deaths in the United States in 2017 [1, 2]. The diagnosis of prostate cancer is obtained by biopsy after clinical, biochemical or imaging suspicion arises [2]. Staging is determined by histology and using imaging modalities, mainly CT and MRI. Patients are then stratified into three risk level groups according to their stage, PSA level and Gleason Score (GS). Metastatic prostate cancer has a recognizable pattern of spread, involving mainly regional lymph nodes (predominantly pelvic and para-aortic) and the skeleton (predominantly the spine) [3]. Additional extranodal metastatic sites occur in the lungs and liver [3, 4]. Treatment strategies of prostate cancer are based on the patient's risk group and include watchful waiting, hormonal therapy, radiotherapy, surgical intervention, chemotherapy or a combination of the above.

Prostate-specific membrane antigen (PSMA) is a type II transmembrane protein that acts as a glutamate carboxypeptidase enzyme $[5,6]$ and is a useful target for diagnostic and therapeutic applications in nuclear medicine because of its' high expression in prostate cancer cells. The physiologic biodistribution of radiolabeled PSMA, at present mainly using 68Ga, includes the salivary and lacrimal glands, the small intestine, liver and spleen. It can also be taken up, to a lesser extent, in normal prostate tissue [5, 6]. 68Ga-PSMA is a valuable tool in the assessment and management of advanced prostate cancer patients. However, a wide range of malignancies other than prostate cancer have also been reported to express PSMA as part of tumor neovasculature [7-9] with 68Ga-PSMA avidity described in cases of breast cancer [10], renal cell carcinoma [11], glioblastoma multiforme [12], hepatocellular carcinoma [13], differentiated thyroid cancer [14], colorectal carcinoma [15], non-small cell lung cancer [16] and follicular lymphoma [17]. PSMA uptake has been also reported in a large variety of benign lesions such as retroperitoneal schwannoma [18], desmoid tumor [19], Paget's disease of bone [20], sarcoidosis [21], sub-acute stroke [22] and bone fractures [23, 24]. Uptake in benign processes as well as in the celiac ganglia can mimic a lymph node metastasis $[24,25]$ and can therefore be pitfalls in clinical practice.

The aim of present study was to describe the metastatic patterns and frequency of involved sites of disease in prostate cancer and also to assess the incidence and outline the characteristics of benign Ga68-PSMA avid $\mathrm{PET} / \mathrm{CT}$ findings in a large patient population.

\section{Materials and methods}

\section{Study population}

All 68Ga-PSMA PET/CT studies performed in two academic centers (RHCC and CSMC) over a 24-month period were retrospectively analyzed. In both centers the routine follow-up for patients with localized disease includes clinical follow-up every 3-6 months with PSA levels tested twice a year and digital rectal examination performed once a year. When PSA values increase, 68Ga-PSMA PET/CT is performed. In patients with metastatic disease, clinical follow-up is performed every 1-3 months including PSA levels and other blood tests. Bone scan and CT are performed every 3-6 months. 68Ga-PSMA PET/CT is performed when either radio ligand therapy using lutetium-177 PSMA or radiation therapy are considered. Patients' charts were extracted from the institutional database and were reviewed. The ethics committees of both centers approved this retrospective data analysis and patient consent has been waived. The following clinical data were retrieved and recorded: age, indication for 68Ga-PSMA PET/CT imaging, GS and PSA level at the time of diagnosis and at the time of the PET/CT study. Previous therapy administered prior to the PET/CT study was also recorded.

\section{PET/CT acquisition and processing}

PET and contrast enhanced CT (when not contraindicated) were acquired consecutively from head to the mid-thigh using a PET/CT system (Discovery 690, GE Healthcare, Milwaukee, US or Gemini XL, Philips Medical Systems, Cleveland, OH, US), approximately $60 \mathrm{~min}$ after the injection on average of $159 \mathrm{MBq}(4.3 \mathrm{mCi})$ 68Ga-PSMA (range: 74 to $219.4 \mathrm{MBq}, 2$ to $5.9 \mathrm{mCi}$ ).

The following parameters were used for CT imaging: pitch $1.375: 1$, gantry rotation time $0.7 \mathrm{~s}, 120 \mathrm{kVp}$, automatically adjusted current in the range $100-650 \mathrm{~mA}$, and a $2.5 \mathrm{~mm}$ slice thickness. A contrast enhanced CT scan was obtained $60 \mathrm{~s}$ after injection of $2 \mathrm{~mL} / \mathrm{kg}$ of non-ionic contrast (Omnipaque 300; GE Healthcare). A PET scan followed in 3D acquisition mode for the same axial coverage. CT images were used for fusion with the PET data. PET images were reconstructed with $\mathrm{CT}$ attenuation correction using a 3D ordered subset expectation maximization (3D-OSEM) or a line of response row-action maximum-likelihood (LORRAMLA) algorithm.

\section{Interpretation and analysis of PET/CT images}

All studies were reviewed retrospectively with knowledge of the patient's clinical history and results of previous imaging studies. A team of two Nuclear Medicine physicians or a Nuclear Medicine Physician and a Radiologist interpreted the PET/CT studies in consensus. Any focal 68Ga-PSMA uptake higher than surrounding activity not associated with a known site of physiological uptake and with a corresponding morphological abnormality on CT was considered pathological and suspicious for malignancy. The fraction of 68Ga-PSMA PET/CT studies that were concluded as positive for malignancy was defined as "detectability rate". Any site of incidental 68Ga-PSMA 
uptake considered to represent non-malignant findings was separately documented. The incidence and location of pathological 68Ga-PSMA avid foci, suspicious to represent malignancy, as well as those of unexpected benign foci of increased 68Ga-PSMA activity were documented and analyzed. Findings were characterized as malignant or as benign based on clinical correlation and other imaging modalities, when available.

\section{Statistical analysis}

Differences between average PSA levels in the study groups were assessed using the parametric Mann-Whitney test. Difference in detectability rates as well as in disease distribution between patient groups, categorized according to referral indications, were assessed using the Chi square and Fisher tests. $P$ value smaller then 0.05 was considered statistically significant.

\section{Results}

Four hundred and forty-five 68Ga-PSMA studies were performed in 438 men (mean age 72.4, range 51-92 years) with prostate cancer. Average GS was 7.5 (range 5-10). Average PSA level at diagnosis was $46.9 \mathrm{ng} / \mathrm{mL}$ (range 0-4000, median 11.0) and at the time of the PET/CT study $18.4 \mathrm{ng} / \mathrm{mL}$ (range $0.05-533$, median 4.3 ). The indications for 68Ga-PSMA PET/CT included biochemical failure ( $n=270,61 \%)$, staging of high-risk disease $(n=112$, $25 \%)$, assessment of response to anti-cancer therapy $(n=30,7 \%)$, follow-up with no evidence of clinical, biochemical or imaging suspicious for recurrence on ( $n=22,5 \%)$ and suspected bone metastases on other imaging modalities performed as routine assessment $(n=11,2 \%)$ (Table 1). Previous therapy, administered before PET/CT, is detailed in Table 1.

68Ga-PSMA avid sites of disease were detected in 319 studies (72\%). Prostate gland involvement was detected in 193 studies (43\%), loco-regional spread including seminal vesicles, bladder, rectum and adjacent fat tissue was seen in 51 studies (11\%). Abdomino-pelvic nodal metastases were found in 129 studies (29\%) and distant metastases including lymph nodes outside the abdomen and pelvis, bones and distant organs in 158 studies (36\%) (Table 2). Radiotracer avidity (SUVmax) for different malignant sites is summarized in Table 2 and Fig. 1.

68Ga-PSMA avid bone metastases were diagnosed in 111 studies (25\%) including oligometastases (up to three lesions, $n=63,57 \%$ ) and multiple metastases (more than three lesions, $n=48,43 \%$ ).

Fifty-five 68Ga-PSMA avid lymph node metastases outside the abdomen and pelvis were identified in 29 studies (7\%), including the mediastinum $(n=25,45 \%)$, the cervical, supra- and infra-clavicular regions $(n=17,31 \%)$, the axillae $(n=4,7 \%)$ and additional thoracic sites (retro-pectoral, internal mammary, retro-crural; $n=9,16 \%)$.
Table 1 Patient Characteristics, $n=445$

\begin{tabular}{ll}
\hline Parameter & Value \\
\hline Age & 72.4 years (51-92 years) \\
PSA & \\
At diagnosis & $\begin{array}{l}46.9 \mathrm{ng} / \mathrm{ml}(0-4000 \mathrm{ng} / \mathrm{ml}, \\
\text { median } 11.0)\end{array}$ \\
& $18.4 \mathrm{ng} / \mathrm{ml}(0.05-533 \mathrm{ng} / \mathrm{ml}$, \\
At time of 68Ga-PSMA PET/CT & median 4.3) \\
Gleason Score & \\
$\leq 6$ & $50(11.4 \%)$ \\
7 & $128(29 \%)$ \\
$\geq 8$ & $146(33 \%)$ \\
Average & 7.5 \\
Therapy prior to 68Ga-PSMA PET/CT & \\
Radical Prostatectomy & $150(34 \%)$ \\
Radiotherapy/Brachytherapy & $171(38 \%)$ \\
Hormonal & $206(46 \%)$ \\
Chemotherapy & $23(5 \%)$ \\
Other & $27(6 \%)$ \\
No prior treatment & $122(28 \%)$ \\
Indication for PET/CT & $270(61 \%)$ \\
Biochemical failure & $112(25 \%)$ \\
Staging -high risk & $30(7 \%)$ \\
Assess response to treatment & $22(5 \%)$ \\
Follow up & $11(2 \%)$ \\
Suspected bone metastases & \\
\hline & \\
&
\end{tabular}

68Ga-PSMA avid metastases in other organs (21 lesions) were observed in 18 studies (4\%). The distribution of these foci included the lungs $(n=10,48 \%)$, liver $(n=5$, $21 \%)$, brain $(n=2,10 \%)$, pleura $(n=2,10 \%)$, spleen $(n=1)$ and peritoneum $(\mathrm{n}=1)$.

According to referral indications, none of the metastases in distant organs were found in patients evaluated at staging, whereas two thirds of these lesions occurred in patients who were investigated for biochemical failure $(14 / 21, p<0.013) .7$ lesions were found in studies done for other indications (assess response to treatment, follow up and suspected bone metastases). There was no statistically significant difference between these groups in other sites of disease involvement including loco-regional spread (14\% in staging vs. $10 \%$ in biochemical failure), abdomino-pelvic nodal metastases $32 \%$ vs. 30\%), bone metastases (19\% vs. $24 \%)$ and distant lymph nodes (4\% vs. $7 \%$ ).

The average PSA level in patients with disease limited to the prostate gland was $17.2 \mathrm{ng} / \mathrm{mL}$ compared to $28.9 \mathrm{ng} / \mathrm{mL}$ in patients with local or distant metastases $(p=0.2)$. The average PSA level in patients who were referred for staging of high risk disease was $29.9 \mathrm{ng} / \mathrm{mL}$ 
Table 2 Distribution of 68Ga-PSMA avid sites of prostate cancer involvement

\begin{tabular}{lll}
\hline & No studies (\%) & SUVmax (range) \\
\hline$N=319$ & $72 \%$ & \\
Prostate & $193(43 \%)$ & $11.3(2-61)$ \\
Loco-regional spread & $51(11 \%)$ & $13(2-78)$ \\
Abdomino-pelvic nodal metastases & $129(29 \%)$ & $12(1.4-100)$ \\
Distant metastases & $158(36 \%)$ & \\
$\quad$ Bone metastases & $111(25 \%)$ & $12.5(1.9-91)$ \\
\multicolumn{1}{c}{ Distant nodes } & $29(7 \%)$ & $11.3(1.5-58)$ \\
Other $^{\text {a }}$ & $18(4 \%)$ & $7.1(1.8-14.6)$ \\
\hline
\end{tabular}

${ }^{\mathrm{a}} 68 \mathrm{Ga}-\mathrm{PSMA}$ avid metastases in lungs $(n=10,48 \%)$, liver $(n=5,21 \%)$, brain ( $n$ $=2,10 \%)$, pleura $(n=2,10 \%)$, spleen $(n=1)$ and peritoneum $(n=1)$

compared to $14.8 \mathrm{ng} / \mathrm{mL}$ in patients with biochemical failure ( $p=0.05$, borderline significant).

Detectability rates of active disease using 68Ga-PSMA were calculated for different PSA levels and according to study indications. The detection rate was $31 \%$ for PSA 0$0.99 \mathrm{ng} / \mathrm{mL}, 63 \%$ for $1-1.99 \mathrm{ng} / \mathrm{mL}, 74 \%$ for $2-3.99,77 \%$ for 3-9.99 and $90 \%$ in patients with PSA higher than $10 \mathrm{ng} / \mathrm{mL}$. In 270 studies performed for the assessment of biochemical failure there were $181(67 \%)$ positive 68GaPSMA-PET/CT studies. In patients referred for staging of high risk disease 94 out of 112 studies (84\%) were positive. The difference in detectability rates between these two patient groups was statistically significant $(p<0.05)$. Detectability rates of malignancy in the additional patient groups were of $73 \%(n=22)$ in cases assessed for monitoring response to treatment, $45 \%$ in patients were referred for follow up $(n=10)$, and $45 \%$ in patients with suspected bone metastases $(n=5)$ (Fig. 2). Due to small patient numbers in these subgroups the level of statistical significance could not be calculated.
Ninety-six 68Ga-PSMA avid foci were categorized as non-malignant in 81 studies (18\%). They were localized to benign reactive lymph nodes $(n=36,38 \%$, in the axilla, inguinal region and pulmonary hila), the skeleton $(n=21,22 \%$, including 7 fractures, 13 degenerative changes and one patient with diffuse bone uptake related to known anemia), the thyroid gland $(n=9,9 \%$, including 6 with focal uptake in thyroid nodules and 3 with diffuse thyroid uptake), lungs $(n=8,8 \%$, including 6 opacities representing inflammatory infiltrates and 2 lung nodules), ganglions $(n=9,9 \%$, including 7 in celiac ganglion, one in stellate ganglion and one in a trigeminal ganglion), gallbladder ( $n=5,5 \%$, without any specific CT findings), stomach or gastro-esophageal region $(n=4,4 \%)$, pancreas $(n=2,2 \%)$ and one case each in an accessory spleen and a surgical scar. Radiotracer avidity (SUVmax) of the nonmalignant sites is summarized in Table 3 and Fig. 3.

\section{Discussion}

68Ga-PSMA PET/CT has an important role in assessment of prostate cancer patients with biochemical recurrence [6, 26-31] and is evolving in staging high- and intermediate risk disease prior to surgery or radiotherapy $[28,32,33]$.

In present study we have assessed the distribution of 68Ga-PSMA avid prostate cancer metastases in a large group of 445 patients from two tertiary medical centers referred mainly for biochemical recurrence $(61 \%$ of patients) and staging of high grade disease (25\%). The highest detectability rates of active disease were observed in these two groups of patients, $67 \%$ and $84 \%$ respectively. In a meta-analysis including 16 manuscripts and 1309 patients, the overall percentage of positive 68Ga-PSMA studies in biochemical recurrence was 76\% (25), increasing from $42 \%$ in patients with PSA levels

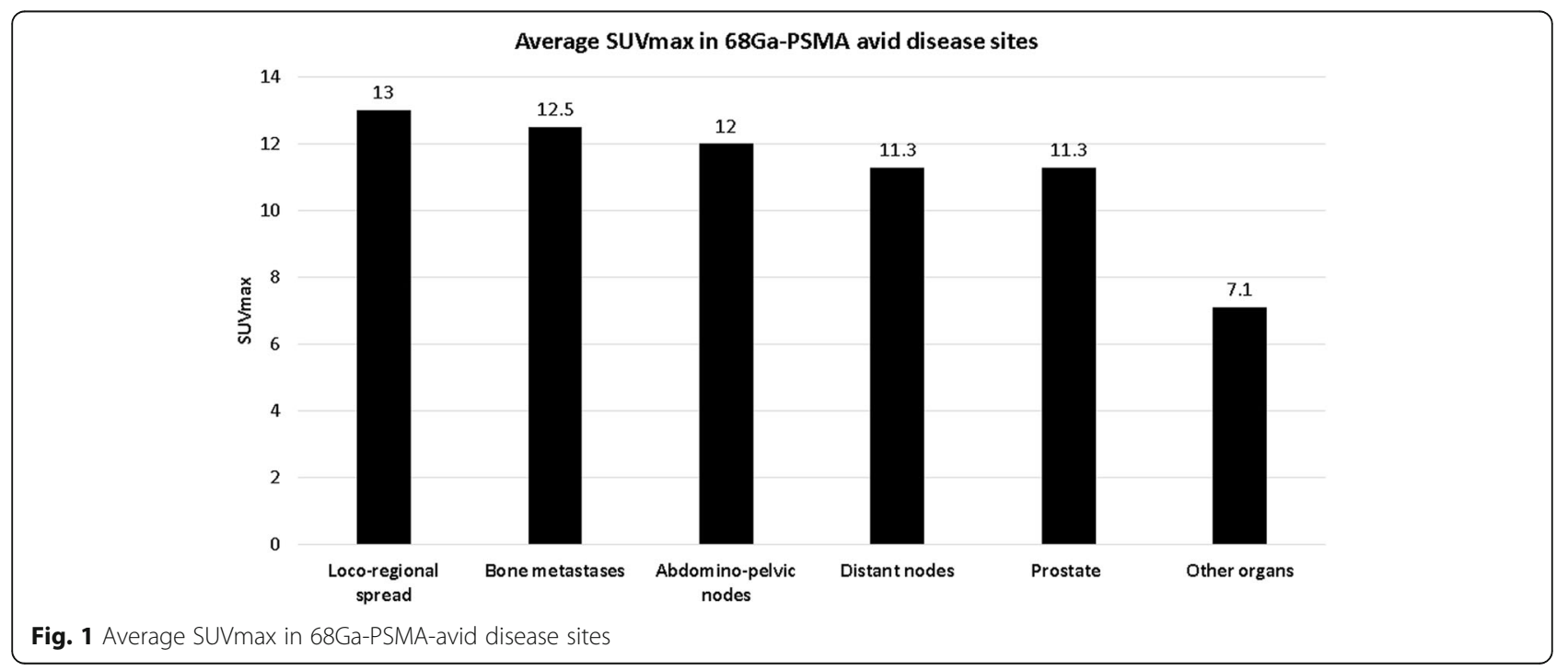




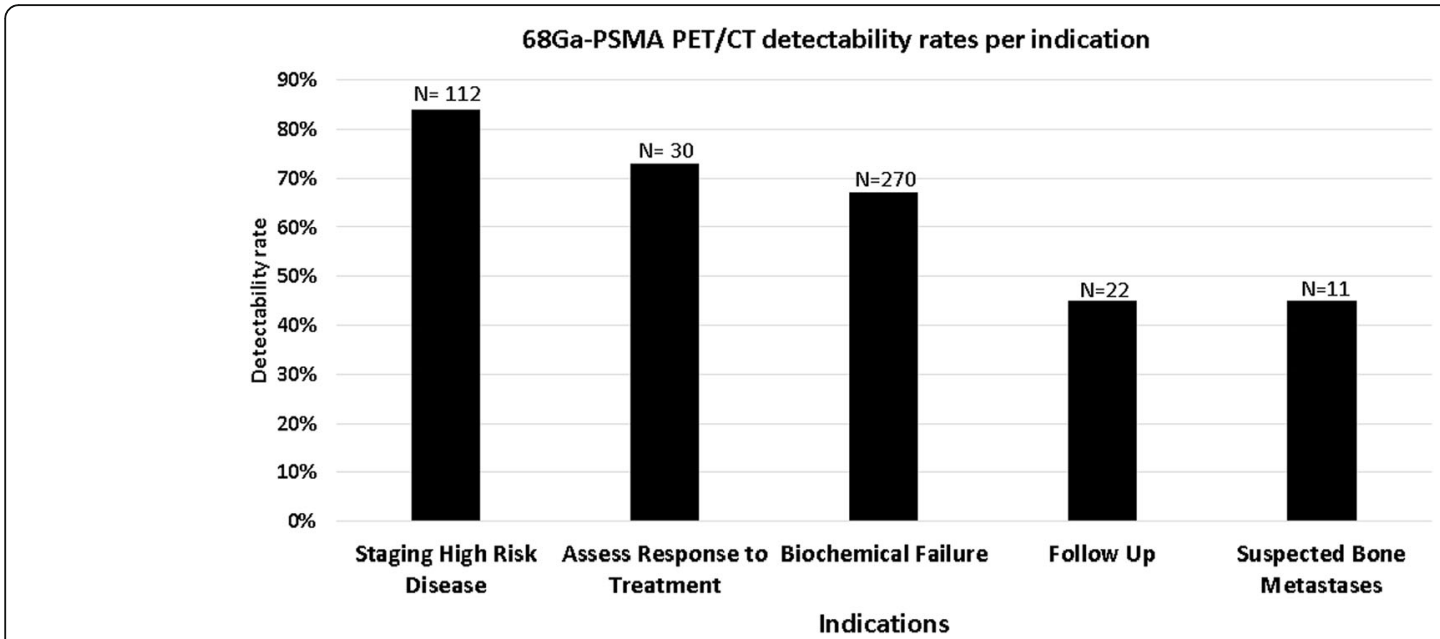

Fig. 2 68Ga-PSMA PET/CT detectability rates per indication

less than 0.2 to $95 \%$ when PSA was greater than $2 \mathrm{ng} /$ $\mathrm{mL}$ (25). Detection rate of $67 \%$ in present study is comparable, taking into account the wide range of PSA values in the study population $(0.05-533 \mathrm{ng} / \mathrm{mL})$. In staging of high risk disease $93 \%$ of patients had intra-prostatic avidity whereas 68Ga-PSMA avid pelvic lymph node metastases were identified in $33.3 \% 4-20 \mathrm{~mm}$ in diameter. There were $66.7 \%$ false negative lymph nodes, measuring 1-10.8 $\mathrm{mm}$ in diameter (30). Another study in 51 patients with high risk disease reported sensitivity, specificity and accuracy of $53 \%, 86 \%$ and $76 \%$ in detecting lymph node metastases. Maximum length of tumor within the detected lymph node metastases was 5-30 mm,

Table 3 Distribution of 68Ga-PSMA-avid non-malignant findings

\begin{tabular}{|c|c|c|}
\hline$N=96$ & No (\%) & SUVmax (range) \\
\hline Benign lymph nodes & $36(38 \%)$ & $2.5(0.7-17.7)$ \\
\hline $\begin{array}{l}\text { Skeletal } \\
\text { Fracture } \\
\text { Degenerative } \\
\text { Anemia }\end{array}$ & $\begin{array}{l}21(22 \%) \\
7 \\
13 \\
1\end{array}$ & $3(1.4-5.5)$ \\
\hline $\begin{array}{l}\text { Thyroid } \\
\text { Focal (nodules) } \\
\text { Diffuse }\end{array}$ & $\begin{array}{l}9(9 \%) \\
6 \\
3\end{array}$ & $4(2.3-9)$ \\
\hline $\begin{array}{l}\text { Lungs } \\
\text { Inflammatory infiltrates } \\
\text { Nodules }\end{array}$ & $\begin{array}{l}8(8 \%) \\
6 \\
2\end{array}$ & $2.6(1.5-4.3)$ \\
\hline $\begin{array}{l}\text { Ganglions } \\
\text { Celiac } \\
\text { Other }^{a}\end{array}$ & $\begin{array}{l}9(9 \%) \\
7 \\
2\end{array}$ & $4.1(2.2-7.4)$ \\
\hline Gallbladder & $5(5 \%)$ & $6.7(5.8-8)$ \\
\hline Stomach or GEJ & $4(4 \%)$ & $3.2(3-3.76)$ \\
\hline Pancreas & $2(2 \%)$ & $2.8(2.7-2.9)$ \\
\hline Accessory spleen & 1 & 4.1 \\
\hline Surgical scar & 1 & 3.3 \\
\hline \multicolumn{3}{|c|}{ aOne stellate ganglion, one trigeminal ganglion } \\
\hline
\end{tabular}

compared to $0.2-8 \mathrm{~mm}$ for undetected involved lymph nodes [34]. In another study in 42 patients with intermediate to high risk prostate cancer 68Ga-PSMA PET/CT identified all 41 involved lymph nodes with a short axis diameter $>10 \mathrm{~mm}$ as well as $8 / 10$ involved lymph nodes with a short axis diameter of $5-10 \mathrm{~mm}$. There were no involved lymph nodes with short axis diameter $<5 \mathrm{~mm}$ [35]. In other studies, neither extrapelvic lymph node metastases nor visceral involvement was found [33, 34]. Similarly, in present study there were no distant $68 \mathrm{Ga}$ PSMA avid metastases in patients referred for staging of high risk disease.

Although the present study population differs, the distribution of metastatic lesions bears some resemblance to data previously published mainly from autopsy [3]. Bubendorf et al. have assessed the metastatic spread of prostate cancer in an autopsy study of 1589 patients [3], about half of them with previously known prostate cancer and the rest with an occult tumor. Metastatic prostate cancer was diagnosed in $35 \%$ of patients. In patients with lymph node metastases paraaortic nodes (in 80\%) and pelvic lymph node metastases (55\%) were most common, followed by mediastinal (40\%) and inguinal nodes (18\%) with only rare involvement of other nodal sites. Bone metastases, predominantly in the spine, were present in $90 \%$ of patients with metastatic disease, $46 \%$ had lung metastases, $25 \%$ liver and $21 \%$ pleural metastases. Rare sites of metastatic disease included the adrenals in $13 \%$, peritoneum in $7 \%$, meninges in $6 \%$, kidney and ureter/urethra $3 \%$ each, pericardium and spleen $2 \%$ each and brain, thyroid, bowel, pancreas and mesentery in 1\% each (3). In present study overall $72 \%$ of patients who were referred for various indications, all with known prostate cancer had 68Ga-PSMA avid disease. The most common sites of 68Ga-PSMA avid metastases included abdominopelvic nodal metastases in $29 \%$, skeletal metastases in $25 \%$, 


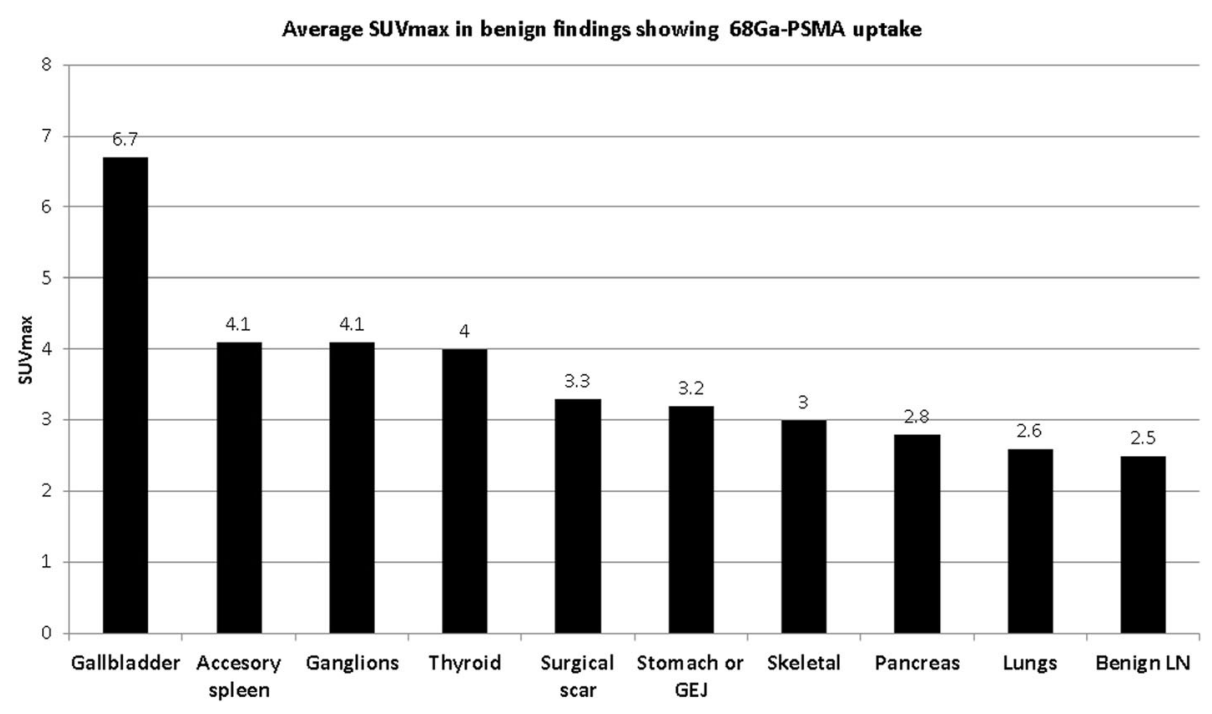

Fig. 3 Average SUVmax in benign findings showing 68ga-PSMA uptake

loco-regional spread in $11 \%$ and distant nodal metastases in $7 \%$ of cases. The most common distant nodal metastases were observed in the mediastinum, followed by cervical, supra- and infra-clavicular regions, with other nodal sites being less commonly involved. Other less common sites of 68Ga-PSMA avid metastases occurred in $4 \%$ of the studies, with almost half of them in the lungs, about $20 \%$ in the liver, and isolated cases of brain, pleura, spleen and peritoneal metastases. Interestingly, distant spread was most frequently seen in patients referred for the assessment of biochemical recurrence, but absent in patients referred for staging of high risk disease.

In present study 68Ga-PSMA avid bone metastases were diagnosed in 111 studies (25\%). Focal 68Ga-PSMA uptake in the skeleton is usually considered to indicate the presence of bone metastases, unless it can be attributed to a corresponding skeletal lesion [18, 20, 23]. In current study, focal 68-Ga-PSMA uptake was also found in a variety of benign bone lesions. Caution and comparison with morphological findings on the CT component are therefore recommended prior to labeling, mainly solitary skeletal lesions, as metastatic. There is limited clinical evidence for the use of 68Ga-PSMA $\mathrm{PET} / \mathrm{CT}$ in the evaluation of bone metastases in patients with prostate cancer. In a recent review of the published literature which included 31 case series and 6 case reports, 68Ga-PSMA PET/CT demonstrated higher diagnostic accuracy than bone scan in the initial staging and in biochemical recurrence, but not in patients with known metastatic prostate cancer [36].

Physiological distribution of 68Ga-PSMA as well as pitfalls and artifacts have been previously reported by several groups [5, 6, 10, 24, 25]. In the early days of using 68Ga-PSMA PET/CT, when the tracer was considered specific for prostate cancer, pitfalls in the interpretation of 68Ga-PSMA PET/CT studies were described, most of them as case reports $[5,17,18,20,21]$, as well as case reports of 68Ga-PSMA avidity in other tumors (9-15).

To provide an accurate interpretation of 68Ga-PSMA PET/CT studies it is therefore important to be knowledgeable of the physiologic distribution of the tracer, the pattern of disease spread, as well as of avidity related to potential benign pitfalls. In present study 96 benign foci of 68Ga-PSMA avidity were found in a subgroup of 81 patients. Following numerous case reports this is, to the best of our knowledge, the first study to assess the prevalence and degree of $68 \mathrm{Ga}$-PSMA avidity of non-malignant findings in a large group of patients with prostate cancer. Benign lymph nodes were the most common site of non-malignant $68 \mathrm{Ga}$-PSMA activity, representing $38 \%$ of foci, followed by skeletal uptake, mainly in degenerative changes and fractures, in $22 \%$. Up to $10 \%$ of findings included focal or diffuse uptake in the thyroid, in lung lesions or ganglions. In present study the prevalence of celiac ganglion uptake was considerably lower (7\%) compared to Krohn et al. who identified focal celiac ganglion activity in 76 of 85 patients (89\%) [25]. Increased gallbladder uptake was seen in 5\% of patients, compared to $10 \%$ previously reported in a series of 40 patients [5].

Although on average the intensity of 68Ga-PSMA uptake in non-malignant findings was significantly lower as compared to that of metastatic lesions, there is a wide range of SUVmax levels. While some of the metastatic lesions presented with SUVmax of 2 or less some of the benign 68Ga-PSMA avid findings showed an SUVmax above 6 . Therefore, it is important to be aware of the possible nonmalignant etiologies of tracer uptake of even moderate or high intensity. 
While in present series no other 68Ga-PSMA avid malignant tumors were identified this tracer has been shown to accumulate in other cancers, specifically in the endothelial cells of tumoral and peri-tumoral capillaries, possibly related to angiogenesis $[8,11-17]$.

The major limitations of present study are its retrospective nature and the lack of histopathological correlative data, the latter compensated by clinical and imaging follow up. However, since this study includes a large patient cohort assessed in two tertiary centers who were referred for different indications present findings could be used as a basis in planning of prospective studies.

\section{Conclusion}

Present study shows 68Ga-PSMA avid sites of disease following the distribution pattern of prostate cancer involvement as previously described mainly in autopsy studies with comparable detection rates. 68Ga-PSMA $\mathrm{PET} / \mathrm{CT}$ is therefore an accurate non-invasive tool. If current results will be confirmed by further prospective studies 68Ga-PSMA PET/CT should be included, in addition to MRI of the pelvis, in the recommendations and society guidelines to evaluate disease extent in patients with prostate cancer.

\section{Abbreviations}

CSMC: Chaim Sheba Medical Center; CT: Computed tomography; Ga: Gallium; GE: General Electrics; OSEM: Ordered Subsets ExpectationMaximization; GS: Gleason score; MRI: Magnetic resonance imaging; PET: Positron emission tomography; PSA: Prostate specific antigen; PSMA: Prostate specific membrane antigen; RHCC: Rambam Health Care Campus; SUV: Standardized Uptake Value

\section{Acknowledgements}

Not applicable.

\section{Funding}

This research did not receive any specific funding.

\section{Availability of data and materials}

The datasets used and/or analyzed during the current study are available from the corresponding author on reasonable request.

\section{Authors' contributions \\ Guarantor of integrity of the entire study: ZK, SBH; study concepts and design: ZK, SBH; literature research: ZK, SBH, RG; data collection: SBH, RG, TD, NP, MM, EG; data analysis: $R G, S B H$; manuscript preparation: $S B H, R G, Z K$, manuscript editing: OI, SBH. All authors read and approved the final manuscript.}

\section{Ethics approval and consent to participate}

Ethical approval was obtained by the local institutional review board.

\section{Consent for publication}

Not applicable.

\section{Competing interests}

The authors declare that they have no competing interests.

\section{Publisher's Note}

Springer Nature remains neutral with regard to jurisdictional claims in published maps and institutional affiliations.

\section{Author details}

'Department of Nuclear Medicine, Rambam Health Care Campus, Haifa, Israel. ${ }^{2}$ The Bruce Rappaport Faculty of Medicine, Technion - Israel Institute of Technology, Haifa, Israel. ${ }^{3}$ Department of Nuclear Medicine, Wolfson Medical Center, Holon, Israel. ${ }^{4}$ Sackler School of Medicine, Tel Aviv University, Tel Aviv, Israel. ${ }^{5}$ Department of Nuclear Medicine, Chaim Sheba Medical Center, Ramat Gan, Israel. ${ }^{6}$ Department of Medical Biophysics and Nuclear Medicine, Hadassah University Hospital, Ein Kerem, Jerusalem, Israel.

${ }^{7}$ University College London and UCL Hospitals, NHS Trust, London, UK.

Received: 30 August 2018 Accepted: 22 October 2018

Published online: 01 November 2018

\section{References}

1. Society AC. Cancer Facts\&Figures: American cancer Societ- Atlanta; 2017.

2. Parker C, Gillessen S, Heidenreich A, Horwich A. Cancer of the prostate: ESMO clinical practice guidelines for diagnosis, treatment and follow-up. Ann Oncol. 2015;26(Suppl 5):v69-77.

3. Bubendorf L, Schopfer A, Wagner U, et al. Metastatic patterns of prostate cancer: an autopsy study of 1,589 patients. Hum Pathol. 2000;31(5):578-83.

4. Vinjamoori $A H$, Jagannathan JP, Shinagare $A B$, et al. Atypical metastases from prostate cancer: 10-year experience at a single institution. AJR Am J Roentgenol. 2012;199(2):367-72.

5. Demirci E, Sahin OE, Ocak M, Akovali B, Nematyazar J, Kabasakal L. Norma distribution pattern and physiological variants of 68Ga-PSMA-11 PET/CT imaging. Nucl Med Commun. 2016;37(11):1169-79.

6. Afshar-Oromieh A, Malcher A, Eder M, et al. PET imaging with a [68Ga] gallium-labelled PSMA ligand for the diagnosis of prostate cancer: biodistribution in humans and first evaluation of tumour lesions. Eur J Nucl Med Mol Imaging. 2013;40(4):486-95.

7. Fragomeni. PSMA-targeted Imaging: Beyond Prostate Cancer PET Center of Excellence Newsletter 2016;13(1).

8. Backhaus P, Noto B, Avramovic N, et al. Targeting PSMA by radioligands in non-prostate disease-current status and future perspectives. Eur J Nucl Med Mol Imaging. 2018:45(5):860-77.

9. Salas Fragomeni RA, Amir T, Sheikhbahaei S, Harvey SC, Javadi MS, Solnes $\mathrm{LB}$, et al. Imaging of nonprostate cancers using PSMA-targeted radiotracers: rationale, current state of the field, and a Call to Arms. J Nucl Med. 2018; 59(6):871-7.

10. Sathekge M, Modiselle $M$, Vorster M, et al. (68) Ga-PSMA imaging of metastatic breast cancer. Eur J Nucl Med Mol Imaging. 2015:42(9):1482-3.

11. Demirci E, Ocak M, Kabasakal L, et al. (68) Ga-PSMA PET/CT imaging of metastatic clear cell renal cell carcinoma. Eur J Nucl Med Mol Imaging. 2014;41(7):1461-2.

12. Schwenck J, Tabatabai G, Skardelly M, et al. In vivo visualization of prostatespecific membrane antigen in glioblastoma. Eur J Nucl Med Mol Imaging. 2015:42(1):170-1.

13. Sasikumar A, Joy A, Nanabala R, Pillai MR, Thomas B, Vikraman KR. (68) GaPSMA PET/CT imaging in primary hepatocellular carcinoma. Eur J Nucl Med Mol Imaging. 2016:43(4):795-6.

14. Verburg FA, Krohn T, Heinzel A, Mottaghy FM, Behrendt FF. First evidence of PSMA expression in differentiated thyroid cancer using [(6)(8) Ga]PSMAHBED-CC PET/CT. Eur J Nucl Med Mol Imaging. 2015;42(10):1622-3.

15. Huang YT, Fong W, Thomas P. Rectal Carcinoma on 68Ga-PSMA PET/CT. Clin Nucl Med. 2016;41(3):e167-8.

16. Shetty D, Loh H, Bui C, Mansberg R, Stevanovic A. Elevated 68Ga prostatespecific membrane antigen activity in metastatic non-small cell lung Cancer Clin Nucl Med. 2016:41(5):414-6.

17. Kanthan GL, Coyle L, Kneebone A, Schembri GP, Hsiao E. Follicular lymphoma showing avid uptake on 68Ga PSMA-HBED-CC PET/CT. Clin Nucl Med. 2016;41(6):500-1.

18. Rischpler C, Maurer T, Schwaiger M, Eiber M. Intense PSMA-expression using (68) Ga-PSMA PET/CT in a paravertebral schwannoma mimicking prostate cancer metastasis. Eur J Nucl Med Mol Imaging. 2016;43(1): 193-4.

19. Kanthan GL, Hsiao E, Kneebone A, Eade T, Schembri GP. Desmoid tumor showing intense uptake on 68Ga PSMA-HBED-CC PET/CT. Clin Nucl Med. 2016;41(6):508-9.

20. Artigas C, Alexiou J, Garcia C, et al. Paget bone disease demonstrated on (68) Ga-PSMA ligand PET/CT. Eur J Nucl Med Mol Imaging. 2016;43(1):195-6. 
21. Kobe C, Maintz D, Fischer T, Drzezga A, Chang DH. Prostate-Specific Membrane Antigen PET/CT in Splenic Sarcoidosis. Clin Nucl Med. 2015; 40(11):897-8.

22. Noto B, Vrachimis A, Schafers M, Stegger L, Rahbar K. Subacute stroke mimicking cerebral metastasis in 68Ga-PSMA-HBED-CC PET/CT. Clin Nuc Med. 2016;41(10):e449-51.

23. Gykiere $\mathrm{P}$, Goethals L, Everaert $\mathrm{H}$. Healing sacral fracture masquerading as metastatic bone disease on a 68Ga-PSMA PET/CT. Clin Nucl Med. 2016:41(7):e346-7.

24. Lambertini A, Castellucci P, Farolfi A, et al. Pictorial essay: normal variants, lesions, and pitfalls in 68Ga-PSMA PET imaging of prostate cancer. Clin Transl Imaging. 2018;6(3):239-47.

25. Krohn T, Verburg FA, Pufe T, et al. [(68) Ga]-PSMA-HBED uptake mimicking lymph node metastasis in coeliac ganglia: an important pitfall in clinical practice. Eur J Nucl Med Mol Imaging. 2015:42(2):210-4.

26. Eiber M, Maurer T, Souvatzoglou M, et al. Evaluation of hybrid (68) Ga-PSMA ligand PET/CT in 248 patients with biochemical recurrence after radical prostatectomy. J Nucl Med. 2015;56(5):668-74

27. Perera M, Papa N, Christidis D, et al. Sensitivity, specificity, and predictors of positive (68) Ga-prostate-specific membrane antigen positron emission tomography in advanced prostate Cancer: a systematic review and metaanalysis. Eur Urol. 2016;70(6):926-37.

28. Roach PJ, Francis R, Emmett L, et al. The impact of (68) Ga-PSMA PET/CT on management intent in prostate Cancer: results of an Australian prospective multicenter study. J Nucl Med. 2018;59(1):82-8.

29. Vinsensia M, Chyoke PL. Hadaschik B, et al. (68) Ga-PSMA PET/CT and volumetric morphology of PET-positive lymph nodes stratified by tumor differentiation of prostate Cancer. J Nucl Med. 2017;58(12):1949-55.

30. Afaq A, Alahmed S, Chen SH, et al. Impact of (68) Ga-prostate-specific membrane antigen PET/CT on prostate Cancer management. J Nucl Med. 2018;59(1):89-92

31. Calais J, Fendler WP, Eiber M, Gartmann J, Chu FI, Nickols NG, et al. Impact of (68) Ga-PSMA-11 PET/CT on the Management of Prostate Cancer Patients with biochemical recurrence. J Nucl Med. 2018;59(3):434-41.

32. Fendler WP, Schmidt DF, Wenter V, et al. 68Ga-PSMA PET/CT detects the location and extent of primary prostate Cancer. J Nucl Med. 2016;57(11):1720-5.

33. Budaus L, Leyh-Bannurah SR, Salomon G, et al. Initial experience of (68) GaPSMA PET/CT imaging in high-risk prostate Cancer patients prior to radical prostatectomy. Eur Urol. 2016;69(3):393-6.

34. Obek C, Doganca T, Demirci E, et al. The accuracy of (68) Ga-PSMA PET/CT in primary lymph node staging in high-risk prostate cancer. Eur J Nucl Med Mol Imaging. 2017:44(11):1806-12.

35. Zhang Q, Zang S, Zhang C, et al. Comparison of (68) Ga-PSMA-11 PET-CT with $\mathrm{mpMRI}$ for preoperative lymph node staging in patients with intermediate to high-risk prostate cancer. J Transl Med. 2017;15(1):230.

36. Zacho HD, Nielsen JB, Haberkorn U, Stenholt L, Petersen L. (68) Ga-PSMA $\mathrm{PET} / \mathrm{CT}$ for the detection of bone metastases in prostate cancer: a systematic review of the published literature. Clin Physiol Funct Imaging. 2018;38(6):911-22.

Ready to submit your research? Choose BMC and benefit from:

- fast, convenient online submission

- thorough peer review by experienced researchers in your field

- rapid publication on acceptance

- support for research data, including large and complex data types

- gold Open Access which fosters wider collaboration and increased citations

- maximum visibility for your research: over $100 \mathrm{M}$ website views per year

At $\mathrm{BMC}$, research is always in progress.

Learn more biomedcentral.com/submissions 